https://doi.org/10.15407/ujpe65.1.17

I. $\operatorname{LICATA}^{1,2,3}$

${ }^{1}$ ISEM, Institute for Scientific Methodology

(Palermo; e-mail: ignazio.licata3@gmail.com)

2 School of Advanced International Studies

on Theoretical and Non Linear Methodologies of Physics

(Bari, Italy)

${ }^{3}$ International Institute for Applicable Mathematics \& Information Sciences (IIAMIS),

B.M. Birla Science Centre

(Adarsh Nagar, Hyderabad 500,India)

\title{
QUANTUM MECHANICS
}

INTERPRETATION ON PLANCK SCALE

In the last years, many different primeval quantization theories on the Planck scale have been developed. Their goal is to provide a vacuum model able to ground the research beyond the Standard Model. Despite their goal is quite ambitious and aims toward particle physics, a necessary and notable consequence is we can read Quantum Mechanics from an emergent viewpoint. Different hypotheses on elementary cells are possible. We will focus here on the conceptual features of $G$. 't Hooft and $F$. Winterberg theories with a special attention for the emerging of non-local correlations. These theories define a new style in the interpretation of Quantum Mechanics.

Keywords: quantum mechanics interpretation, Planck scale, 't Hooft equivalence class, Winterberg plasma, non-locality.

\section{Motivations: Deconstructing Bell}

The idea that a more subtle level of the discrete deterministic type may exist under quantum mechanics (QM) is as old as the Bohr-Einstein debate (1935). As a conceptual scheme, it goes back to the successes of statistical physics. More than half a century of interpretative debates has a limited confidence in the Copenhagen interpretation for APP (All Practical Purposes), but it is not even possible to say that a subquantum model has been clearly established. The contrast between a Bohr-type irreducible non-locality and an objective realism of the Einstein type is continually reproposed and has also given some considerable contribution to the understanding of the structure of quantum theory, in particular, the presence of stochastic aspects. The real problem re-

(C) I. LICATA, 2020

ISSN 2071-0194. Ukr. J. Phys. 2020. Vol. 65, No. 1 mains the gap between this type of foundational debates and the complex structure of elementary particle physics, especially in relation to the concept of "vacuum state" in QFTs (Quantum Field Theories).

In particular, the theories of hidden variables have received a severe blow due to Bell's theorem. The possibility of restoring a scheme of this type, with measurable deviations from QM, is now confined to a very thin margin $[1,2]$. To ask ourselves if this is really the last word on $\mathrm{QM}$ is equal to propose an approach to quantum theory in which the violation of Bell's inequalities is compatible with some form of locality, located in a different range from the one in which EPR-Bell experiments are usually performed. In other words, we need to investigate the possibility of an emerging QM constrained from below on a level, where it is possible to find, at least partially, a structure with discrete and localized in- 
formation independent of the observer. However, we must expect that the observables we work with are usually not beables, but emerging variables. A theory is thus outlined in which the local level is not hidden among the observables, but is instead placed on a structurally different plane. This would clarify the failure of traditional hidden variables and proposes a radically new vision between discrete/continuous and classical/quantum. Theories of this type exist and will be referred to here as theories of primitive quantization or pre-quantization. They work on the Planck scale. There are some rather strong reasons for investigating such a theory. The main ones are surely the Bekenstein principle and the holographic principle, both firmly connected to the constraints on the spectrum of quantum fluctuations of the space-time and ultimately to the homogeneity of the tessellation of the same space-time [3;4]. The main motivations of theories of this type are related to quantum gravity, they are not born as "interpretations of QM", but this is considered a necessary step to eliminate the ambiguities generated by the quantummechanical formalism near the Planck scale and at the cosmological level. In fact, the stochasticity has a mesoscopic flavor, and one would not expect to find it at a scale of radical discretization. Ambiguities do not decrease with the second quantization, but increase; in general and on all scales, the elegance of the description of physical processes through amplitudes [5] is strongly limited by the probabilistic mortgage. The latter should derive from deeper reasons, and the Born rule should emerge as a sort of "regulator" of the collective dynamics and their degrees of freedom.

In general, a theory of primordial quantization is based on some general assumptions:

a) Nature works in a similar way to a computer on the Planck scale. This implies that information at that level is finite, local, independent of the observer, and pre-deterministic. Therefore, no interference figures or overlaps are possible, there are very precise rules that assure the transitions from one cell to another through a discrete sequence of states. In practice we are doing a kind of classical physics on the Planck cells. We will call the magnitudes of this level, following 't Hooft, beables, in honor of J.S Bell. Beables are carriers of defined properties and, therefore, form a set of operators that always switch. b) The type of theory depends on the assumptions made on ccell dynamics. The theories that we review here, by 't Hooft and Wintenberg, are quite different also as cultural milieu, but both suggest that the introduction of non-linear oscillators together with the very powerful hypothesis of primordial discretization allows to "rebuild" QM. An essential point is that the subquantum freedom degrees are equal and indistinguishable. In this way, they ensure, at the macroscopic level, the isotropy and homogeneity of the space-time;

c) The hypothesis of locality and pre-determinism directly leads to that of reversibility. At the beables level, information is never lost. However, this does not mean that information on a single cell is recoverable; indeed, it is precisely the loss and selection of information at the emerging level that leads to the observable non-commutative notes of $\mathrm{QM}$, the changeables and the relative equivalence classes of 't Hooft. A quantum state is therefore a derivative concept and can show strong non-local correlations related to the loss of information, which finally acquire an authentic statistical meaning. If the natural constraint on the speed limit of light is introduced onto the lattice, the maximal acceleration of Caianello is obtained [6].

The bet on the emergence of these theories lies therefore in proposing an effective mechanism able to bridge the gap between the Planck scale $\left(10^{19} \mathrm{GeV}\right)$ and the usual one of experiments involving a quantum-mechanical treatment $\left(10^{3} \mathrm{GeV}\right)$. This depends critically on the choice of beables and the relationship between beables, Planck cells, and changeables. So, the primordial quantization theories do not tend to give a "realistic" character to the wave function or even to predict deviations from the standard QM. Their character is rather explanatory of the nature of the QM. Please note that the Planck cell is the extreme limit of the causal connection with the laboratory [7], and the point of arrival is the "ordinary" QM. Therefore, the physically significant aspect consists in the exploration of physical processes in that middle ground that allows the passage from the deterministic and local systems of the beables to quantum behaviors with their typical uncertainty and non-local "weirdness".

To understand how the goal of these theories is necessarily more ambitious, let us think of a classic problem of QM, the so-called "collapse" problem, and 
let us look at it in relation to particle physics. The anticommutative nature of the observables tells us that the result will belong to the changeables class, but we know that a particle like the electron has well-defined characteristics, always, as a charge, mass, spin, likebeables. The aim of the pre-quantization theories is therefore to merge QM and QFT by defining a sort of informative pre-QFT on the Planck scale, thus realizing the "it from bit" principle [8]. In the following, we will limit ourselves to the emergence of QM, starting from the structural links between QM and emerging systems.

\section{Quantum Mechanics as Contextual Theory of Systems}

In recent years, the study of the physics of the emergency has known new developments toward a general theory of great systemic and epistemological interest for the understanding of the structure of physical theories (for a varied general approach, see [9]). It is significant, and not accidental, that the quantum formalism has proved extremely fruitful and powerful to describe emerging and contextual systems in areas far removed from physics. This suggests something about the very nature of QM $[10,11,12,13,14]$.

A system can show contextual aspects in relation to: 1) components; 2) measurement operations; 3 ) system/environment relations. Moreover, a system is said to be emerging, when there are at least two levels, and the following conditions occur: i) the lowlevel description is necessary for a complete understanding of the upper level, but: (ii) the lower level (generally "with arrows pointing at the components", to say it with Weinberg) is not necessary for the description of the upper level [15]. The radical emergency aspects are therefore connected to the fact that, despite the intimate connection, the upper level appears autonomous both from the formal and experimental points of view and can show unprecedented properties at the most subtle level [16]. The relative autonomy of the two levels can also take the form of a different semantics, as traditionally occurs between consciousness and its neural correlates. In this last example, however, it is this autonomy that makes it more reductive to reduce the problem of "free-will" in merely neuronal terms.

Here, it seems to echo the debate between Einstein and Bohr on the completeness of QM, which can therefore be assumed to be primary and fundamental at a level - it is the case of theories that introduce the non-locality $a b$ initio -, but which does not exclude a local and deterministic level, where the characteristics of QM are emerging and find their full meaning. It is the case of the relationship between beables and changeables.

In general, a model, in which the state variables and their transition laws are always defined and acquirable (indifference with respect to the measure), is called logically or informationally closed and is deterministic and local. It is the case, for example, of classical physics, where the measurement operations on all the state variables commute and are simultaneously compatible, i.e., all measuring devices related to different variables can always be used without interfering with one another and without any loss of mutual information. The logic of classical physics is therefore a Boolean-like orthocomplemented logic, which formalizes the possibility of acquiring complete information on every state of the system for every time interval. Information is here understood in the Shannon-Turing sense. At first glance, it would seem that systems of this type have little chance of producing authentic emergencies, but we know from the study of dynamical systems that it is not so. Not only is there not necessarily a correlation between local and global predictabilities. But, thanks to non-linearity, systems of this type can be exponential information amplifiers. For discrete systems such as Cellular Automata (CA), the Langton-Wolfram class IV, dynamic typology capable of exhibiting a strongly peculiar mixture of order-chaos, i.e., on the one hand, to memorize information for long periods, on the other, to exhibit dissipative and chaotic aspects. This may suggest that these are systems capable of emergency on the margins of chaos in a peculiar way with respect to the continuous case. In the case of beables, the informational closure with respect to the observer must be understood in the most drastic way, if these are placed on the Planck scale.

The situation for QM is different. In fact, the principle of indifference of the state of a system with respect to the observer falls, which leads to the collapse of local causal determinism. This aspect is expressed in the formalism of QM by the fact that a closing relationship is only valid for the eigenstates and naturally leads to a characterization of quantum systems as open systems with respect to the observer. This in- 
formation gap can be filled in various ways (hidden variables, superluminality, some kind of non-local information such as the active information of Bohm and Hiley $[17,18])$, but it fixes the contextual aspect of the model characterized by anticommutativity relations. This informational openness to the observer is a general feature of complex systems and is a strong indication of the emergent nature of QM [19, 20, 21].

Another interesting theme, decidedly pre-mature at the time of the historical formation of QM, is the exploration of non-linearity and the new articulations of the KAM theorem [22]. In particular, we now know the effects of non-local correlations described by global informational measures such as Tsallis entropy and Fisher's information, which suggest something more than a mere formal analogy and, at the same time, naturally clarify similarities with stochastic systems $[23,24,25,26]$. Indeed, this type of reading has allowed us to understand why classical systems, however strongly non-linear, can be described so well by an appropriate quantum potential, as happens in many processes modeled through a "neural fluid" [27]. Ultimately, there are strong structural clues that QM is a coarse-grained and emergent description of a subquantum level that, given the failures of traditional theories with hidden variables, should be sought on the Planck scale. The latter should then turn out to be a sort of pre-QFT, so as to weld once and for all the gap between QM and QFT. We now have the reasons to investigate two pre-quantization proposals.

\section{The 't Hooft Approach: \\ Cellular Automata in Hilbert Space}

G. t 'Hooft, one of the physicists who closed the first phase of the standard model (SM) and opened it to the new challenges of quantum gravity, is the author of a simple radical proposal that moves the hidden variables to the Planck scale. In other words, it is not a matter of finding the hidden variables inside the wave function, but rather of considering the latter emerging and bound by a base level, where the beables are located. With an elegance reminiscent of Dirac's style, these are described as a dynamics of cellular automata (CA) written in a Hilbert space. This allows one to introduce the phase linking processes in a simple way and to build, starting from a local and deterministic level, emerging equivalence classes that are the traditionally considered "quantum" behaviors [28-31]. For example, non-local phenomena are an equivalence class constructed from an essentially local substrate. The term "equivalence classes" recalls, not surprisingly, statistical physics and non-linear systems. In fact, it is understood that the same wave function can be produced by several behaviors of the elementary cells. Among the classic observables and beables, there are, in fact, the changeables, i.e., the dynamic emergence processes of quantum phenomena starting from a pre-quantization which is "classical" in many aspects. It is admitted that multiple changeables configurations can give rise to the same observables, just as it happens, for example, with the Wolfram-Langton conjecture and in the more traditional statistical mechanics [32]. In this process, the concept of loss of degrees of freedom is crucial: in the transition from beables to observables, progressively gradual degrees of freedom are lost through the coupling of cells. We leave the declaration of his program to the usual clarity of t'Hooft:

"Quantum mechanics could well relate to microphysics in the same way as thermodynamics relates to molecular physics: it is formally correct, but it may well be possible to devise deterministic laws on the microscale. Why not? The mathematical nature of quantum mechanics does not forbid this, provided that one carefully eliminates the apparent no-go theorems associated to the Bell inequalities. There are ways to re-define particles and fields such that no blatant contradiction arises. One must assume that all macroscopic phenomena such as particle positions, momenta, spins, and energies relate to microscopic variables in the same way as thermodynamic concepts such as entropy and temperature relate to local, mechanical variables. The outcome of these considerations is that particles and their properties are not, or not entirely, real in the ontological sense. The only realities in this theory are the things that happen on the Planck scale. The things we call particles are chaotic oscillations of these Planckian quantities. [33] (...) In our quantum mechanical theories on the atomic scale, we have not yet identified those observables that truly correspond to the actual states of a system. All operators we use on the atomic scale are superpositions of primary observables ('beables') and typical permutation operators ('changeables')" [34].

It must be said that the 't Hooft equivalence classes are a conjecture, albeit fruitful. It is permitted by the 
structure of QM and by the historically consolidated result that each quantum theory admits at least one deterministic/stochastic model, but there is no stringent proof of its validity [35]. Indeed, it is possible to say that precisely the dissipative mechanisms, under which the relationships between beables and observables are hidden through changeables, prevent a demonstration of the conjecture. In a re-presentation of the Bohr-Einstein debate, the last word would belong to Einstein but at the cost of an irreducible dissipation: God's dice are deterministic but very subtle!

The exploration of the 't Hooft conjecture requires a dynamic hypothesis on the cell structure. This is what a very active group of scholars near to the ideas of 't Hooft are doing in these years [36-39]. One starts from the idea that writing a CA in a Hilbert space is equivalent to describe an oscillatory behavior. In this way, a vast program has begun that aims to describe quantum phenomena in terms of classical nonlinear oscillators under pre-quantization constraints. This is a point of extreme importance, since the traditional roles of the classical/quantum levels are practically reversed and mixed. The situation is now more complex and stratified:

3. Classical World (Laplacian determinism by "collapse" and "decoherence" from equivalence classes)

2. Observed Quantum Behavior (quantum observables and non-locality via equivalence classes)

1. Quantized Planck Scale World (deterministic, local CA beables in a Hilbert space; non-linear oscillators?).

The quantum level is therefore "compressed" between a discrete local determinism and a classical and continuous determinism. The questions multiply, because now the question does not consist only in showing the validity of the passage from (1) to (2), but it strongly re-proposes the question of relations between (2) and (3). In particular, at the beables level, there are no overlapping states, but it is difficult to say what the measure consists of. In other words, the question arises of understanding in a single measurement what belongs to the beables and what to the changeables (which can also have a long-term stationarity and permanence). It is presumable that this point can only be clarified with a decisive development of quantum gravity, and it cannot be excluded that the "qualities" that we consider "permanent" (spin, charge, mass, etc.) have an emergent nature in reality. Finally, the connection between the 't

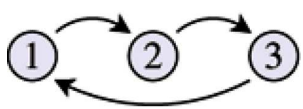

Fig. 1. Three-state universe

Hooft conjecture and the holographic principle must be mentioned. In fact, the conjecture is equivalent to postulating a finite quantity of elementary information for each cell. It may seem strongly anti-intuitive that the informational contribution of a cell $(3 \mathrm{D})$ is measured in terms of area, but different approaches $[40,41]$ showed that this is a consequence of the fact that, on the average, each cell occupies a volume of $l l_{P}^{2}$. Therefore, a spatial region of size $l$ cannot contain more than $l^{3} /\left(l l_{P}^{2}\right)=\left(l / l_{P}\right)^{2}$ cells. This corresponds precisely to the case provided for by the holographic principle, in which the maximum number of information bits stored in a region of size $l$ is $\left(l / l_{P}\right)^{2}$.

A general expression of the reduction in degrees of freedom in an emerging QM model is

$[q, p]=i \hbar\left(1-\frac{E}{E_{p}}\right)= \begin{cases}0, & E+E_{p}, \\ i \hbar, & E \ll E_{p},\end{cases}$

where commutation relations "take shape" for much smaller energies very less than the ones of the Planck scale [42]. In what following, we will refer to the discussion developed in $[37,43,44]$.

Now, we hypothesize a toy model of the Universe with deterministic transitions, i.e., the unitary evolution from one state to another such as: and we write a "formal" wave function for the three states shown in the figure 1:

$|\psi(t)\rangle=\alpha|1\rangle+\beta|2\rangle+\gamma|3\rangle$

The deterministic evolution of this system in the Universe clock will be given by:

$|\psi(t+\tau)\rangle=U(t+\tau, t)|\psi(t)\rangle=\left(\begin{array}{lll}0 & 0 & 1 \\ 1 & 0 & 0 \\ 0 & 1 & 0\end{array}\right)|\psi(t)\rangle$

where the unitary matrix is $U(t+\tau, t)=e^{-i H \tau}$, and the states obey the Born rule:
$P(1)=|\alpha|^{2}$,
$P(2)=|\beta|^{2}$,
$P(3)=|\gamma|^{2}$. 


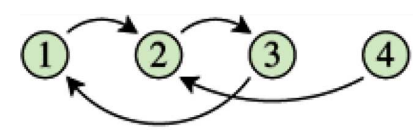

Fig. 2. Four-state universe will non-unitary evolution

Now, let us consider a non-unitary evolution (therefore with an elementary form of dissipation) in a toy model of the type (Fig. 2).

The evolution matrix is:

$U(t+\tau, t)=\left(\begin{array}{llll}0 & 0 & 1 & 0 \\ 1 & 0 & 0 & 0 \\ 0 & 1 & 0 & 0 \\ 0 & 0 & 0 & 0\end{array}\right)$.

The states $\langle 1|$ and $\langle 4|$ "merge" into $\langle 2|$. Thus, the following equivalence classes are defined:

$|1\rangle \equiv\{\mid 1), \mid 4)\}, \quad|2\rangle \equiv \mid 2), \quad|3\rangle \equiv \mid 3)$.

This is the logic that underlies the formation of the equivalence classes through the loss of information (with a dissipative mechanism). Naturally, a realistic case is much more complex, e.g., it could imply a new $\left\langle\left. 2\right|^{*}\right.$ status. As we can see, it is a situation very similar to that in which different chaotic dynamics lead to the same limit cycles. This analogy with non-linear processes returns with greater force in Winterberg's theory.

Let us now consider a traditional Hilbert space with $N$ dimensions and postulate that the wave function of a quantum system can be reproduced by a deterministic system with two degrees of freedom such as

$|\psi\rangle=|\psi(\omega, \phi, t)\rangle$

Let us define the relations between $\omega$ and $\Phi$ :

$\frac{d \phi}{d t}=\omega, \quad \phi \in[0,2 \pi]$

$\frac{d \omega}{d t}=-k f(\omega) f^{\prime}(\omega), \quad k>0$,

$f(\omega)=\operatorname{det}(H-\omega)$,

$f(\omega)=0$,

$\phi$ with period $T=2 \pi / \omega$.

As can easily be seen, the corresponding deterministic system is that of a "material point" which moves on a circle with assigned angular velocity $\omega$ and phase
$\Phi$, and a dissipative element. We introduce two operators whose role is to mediate between classical and quantum languages,

$p_{\phi}=-i \frac{\partial}{\partial \phi}, \quad p_{\omega}=-i \frac{\partial}{\partial \omega}$,

and a temporal rule:

$h=\omega p_{\phi}-\frac{1}{2} k\left[f(\omega) f^{\prime}(\omega), p_{\omega}\right]_{+}$.

In this way, we fix two classical and deterministic equations of the motion:

$$
\begin{aligned}
& \frac{d \phi}{d t}=-i[\phi(t), h] \quad(=\omega), \\
& \frac{d \omega}{d t}=-i[\omega(t), h] \quad\left(=-k f(\omega) f^{\prime}(\omega)\right) .
\end{aligned}
$$

We note here that $h$ is Hermitian, even if the equations of motion are dissipative.

Now, let us consider the Fourier transform of the periodicity in $\Phi$ :

$|\psi(\phi, \omega, t)\rangle=\sum_{n} e^{i n \phi}\left|\psi_{n}(\omega, t)\right\rangle$.

As $\mathrm{t} \rightarrow \infty, \omega(t)$ converges to the fixed point $\omega_{i}$, and the deterministic system tends to

$|\psi(\phi, \omega, t)\rangle \rightarrow \sum_{n} e^{i n\left(\phi-\omega_{i} t\right)}\left|\psi_{n}\left(\omega_{i}, t\right)\right\rangle$

For the eigenstates of the energy, the following equation is therefore valid:

$e^{i E_{i} t^{\prime}}\left|\psi\left(E_{i}\right)\right\rangle=e^{-i n^{\prime} \omega_{i} t}\left|\psi_{n^{\prime}}\left(\omega_{i}, 0\right)\right\rangle \operatorname{con} E_{i}=\omega_{i} !$.

In this way, we obtain a wave function that shows the overlap and interference in the energy eigenstates $E_{i}$ in correspondence with the values of $\omega_{i}$. The phase of this wave function therefore indicates the limit cycle of this state, while the amplitude is the probability that this state will be realized. It should be noted that there is a connection between the zeropoint energy of a quantum oscillator and the geometric phase of a "classical" oscillator. The appearance of a zero-point energy is an important "symptom" of quantization, because it indicates that the commutator of the observables is not zero. It is difficult to underestimate the constructive indications of this elementary model. In fact, it is equivalent to 
saying that the wave function is the "covering" of a large number of coupled elementary oscillators, i.e., "what remains" of their dissipative behavior. This is the meaning of the "Quantization is Dissipation" slogan, which promises to have a formidable impact on quantum gravity researches. In fact, in the latter, we usually start from the famous start with a classical theory, then quantize; if the approach indicated by 't Hooft and his school is correct, this method could be as meaningless as trying to quantize the sound waves in air. Rather we have to go another way, i.e., to find the "right" dissipative mechanism starting from a pre-quantization. The latter is then the characterizing element. In fact, if we limit ourselves to the formal aspects, we obtain a formally equivalent theory but ontologically different as that of the Elementary Cycles of D. Dolce [45]. As regards the non-locality, it is not difficult to program a $\mathrm{CA}$ with non-local behavior (e.g., for two adjacent two-color cells $a \& b$ : For every "discrete instant" $t$, if $a$ is blue, then $b$ is red, and vice versa).

But if the question becomes a bit more physical, as in the model of Planck's "classic" microoscillators, the conclusion is that non-locality is a form of the emerging coherence, or - as critics of 't Hooft say directly taking up the abstract scheme of the $\mathrm{AC}-\mathrm{a}$ form of the superdeterminism: in other words, everything connected to the level of beables is also in the observables, and every free-will choise is only apparent. Although this is the point that most worries the philosophers, it must be said that the objection of physicists is addressed elsewhere. If, on the one hand, it is easy to hypothesize that everything at the time of the Big-Bang was related, it is more difficult to understand how these correlations resist with the dissipation of the emerging levels [46, 47].

\section{Winterberg's Planck Plasma: an Exactly Non-Relativistic Theory}

Friedwardt Winterberg was one of the last pupils of W. Heisenberg. Like many scholars of his generation - for example, our almost contemporary, A. Sakharov, on the opposite side of the world political chessboard - is best known for his research on nuclear fusion, giving theoretical articles at regular intervals starting from the non-linear unified spin theory of his master Heisenberg [48], to arrive in the last years to a theory of pre-quantization, the Planck Plasma The- ory. If 't Hooft can be compared to Dirac's style, Winterberg has something of the essential pragmatism of Schrödinger, and its retrò taste. His ideas about fundamental physics and plasma research - as in the case of D. Bohm [49] and later by R. Laughlin - have a non-trivial connection. In fact, both of them present the idea of a sort of dynamic medium from which particles emerge as solitons or "field globules", to use Einstein's terminology. Moreover, Winterberg's theory is much more classical than that of 't Hooft, at least in its initial formulation, and is exactly non-relativistic, in the sense that even the Lorentz invariance is considered emerging and valid for low energies, an aspect that has preceeded the current forms of double relativity [50]. As we did for 't Hooft, we will give a short summary of the work of Winterberg here, following papers [51-58].

From one of the first titles on the subject, Planck Scale Physics and Newton's Ultimate Objects Conjecture (on Acta Phys. Pol., 27, 10, 1996), it is clear that Winterberg has no embarrassment in recovering the old theories of the ether in a new context. Already L. Janossy had shown that if an ether exists, it is undetectable and, therefore, relativistic, which can also be said of the Planck scale $[59,60]$. In other words, for the purposes of mathematical construction, the basic ontological elusibility is not important, as we have already seen in the case of 't Hooft and other highly speculative approaches of contemporary physics, where sometimes even the boundaries between geometry and physics fade [61]. In particular, the ultimate Newtonian objects, i.e., a mix of positive and negative masses, each one allocated in a Planck cell, was in its initial form the kind of naive hypotheses in contrast with particle physics, despite attempts to show at least the theoretical plausibility of $\mathrm{H}$. Bondi and later by B. Bonnor $[62,63]$. If, instead of a Newtonian "anti-mass," we think in terms of modes of the field, it is rather much easier to accept the idea of a vacuum made of positive and negative modes as in a pre-QFT. The best starting point is probably A. Sakharov's proposal to describe GR as a metric elasticity of vacuum [64, 65], then explicitly postulating the possibility of temporal inversions and fluctuations of the metric at the origin of the Universe (time reversal $=$ negative mode) [66]. The idea of metric fluctuations has been repeated several times [67], and some recent results seem to indicate a) that the temporal inversion is not, as is believed, in contrast 
with the CPT theorem and b) that negative masses are an necessary ingredient in the quantum phase of the Universe (de Sitter model) [68, 69, 70]. Finally, the time reversal plays a key role in many QM readings today [71].

Let us consider thus a Planck Plasma composed by Planck masses $\pm m_{p}$, where each occupies a volume $\pm r_{p}^{3}$. The interaction between the masses is a Planck force $m_{p} c^{2} / r_{p}=c^{4} / G$, where $G$ is the gravitational constant. As we see, the force is a bond a la Hertz, and the Planck length is analogous to Debye length in the plasmas. The interaction between cells is ruled by the fluctuation of the momentum $\Delta p=m_{p} c$ on a length $r_{p}$, so that the Heisenberg relation is not a limit on the precision of measurements, but emerges from the space-time texture itself $\Delta p \Delta q=m_{p} c=\hbar$. The interaction on the Planck scale generates a global dynamics of Zitterbewegung [72, 73] of the type:

$V_{D}=-\left(\frac{r_{p}}{2}\right) \nabla n / n$,

where $V_{D}$ is the diffusion speed and $n$ is the number density of particles. It is well known from the stochastic approaches [74] that the kinetic energy of this diffusive process is given by

$$
\left(\frac{m_{p}}{2}\right) V_{D}^{2}=\left(\frac{m_{p}}{8}\right) r_{p}^{2} c^{2}\left(\frac{\nabla_{n}}{n}\right)^{2}=\left(\hbar^{2} / 8 m_{p}\right)(\nabla n / n)^{2} .
$$

A dynamics of this type is that of particle-hole, well known in condensed matter physics, where the role of the gap is assumed by the negative charge. It is known from the early historical phases of QM that this diffusion is typical of a fluid without viscosity, an analogy linked to the similarity between the Schrödinger equation and the classical diffusion one. This analogy was considered for a long time purely formal and constituted an interpretation of QM based on a superfluid medium. In Winterberg's approach, it springs from a physical dynamics on the Planck scale. Placing

$V=\frac{\hbar}{m_{p}} \nabla S$

where $S$ is the Hamiltonian action, one obtains the Lagrangian

$$
L=n\left[\hbar \frac{\partial S}{\partial t}+\frac{\hbar}{2 m_{p}}(\nabla S)^{2}+U+\frac{\hbar^{2}}{8 m_{p}}\left(\frac{\nabla n}{n}\right)^{2}\right] .
$$

Hence, it is easy to derive the variations of $L$ with respect to $S$ and the density $n$ :

$\frac{\partial n}{\partial t}+\frac{\hbar}{m_{p}} \nabla(n \nabla S)=0$

and

$\hbar \frac{\partial S}{\partial t}+U+\frac{\hbar}{2 m_{p}}(\nabla S)^{2}+\frac{\hbar^{2}}{2 m_{p}} \frac{\nabla^{2} \sqrt{n}}{\sqrt{n}}=0$.

It is easy to "cover up" the collective behaviors of the mix of modes with a wave function thanks to Madelung's transformations:

$$
\left.\begin{array}{l}
\psi=\sqrt{n} e^{i s} \\
\psi^{*}=\sqrt{n} e^{-i s}
\end{array}\right\}
$$

At this point, it is easy to obtain the well-known Schrödinger equation

$i \hbar \frac{\partial \psi}{\partial t}=-\frac{\hbar^{2}}{2 m_{p}} \nabla^{2} \psi+U \psi$

where $U$ is

$U=2 \hbar c r_{p}^{2}\left[\psi_{+}^{*} \psi_{+}-\psi_{-}^{*} \psi_{-}\right]$.

Here, as in 't Hooft, the wave function is emerging from Planck's scale behaviors!

However, the surprises do not end there. To affirm that QM is emerging means to say that a quantum object is not only "driven" by a wave function, but more radically that that system is produced by the same dynamics from which the wave function emerges. This justifies replacing the complex conjugate $\psi_{ \pm}^{*} \psi_{ \pm}$with creation/destruction operators with the commutation relations typical of a proto-QFT:

$$
\begin{aligned}
& {\left[\psi_{ \pm}(\underline{r}) \psi_{ \pm}^{\dagger}\left(\underline{r}^{\prime}\right)=\delta\left(\underline{r}-\underline{r}^{\prime}\right)\right]} \\
& {\left[\psi_{ \pm}(\underline{r}) \psi_{ \pm}\left(\underline{r}^{\prime}\right)\right]=\left[\psi_{ \pm}^{\dagger}(\underline{r}) \psi_{ \pm}^{\dagger}\left(\underline{r}^{\prime}\right)\right] .}
\end{aligned}
$$

At this point, we obtain a non-relativistic equation of the Heisenberg-Pauli-Ivanenko type, one of the first authentically quantum "theories of the Whole" based on non-linear spinors [48]:

$i \hbar \frac{\partial \psi}{\partial t}=\mp \frac{\hbar^{2}}{2 m_{p}} \nabla^{2} \psi_{ \pm} \pm 2 \hbar c r_{p}^{2}\left(\psi_{ \pm}^{\dagger} \psi_{ \pm}-\psi_{\mp}^{\dagger} \psi_{\mp}\right) \psi_{ \pm}$.

ISSN 2071-0194. Ukr. J. Phys. 2020. Vol. 65, No. 1 
The fact that this equation is not relativistic is not a defect here, but rather a virtue; in fact, it ensures that the Hilbert space of state vectors is always positive (i.e., we never observe the gaps). In Winterberg's theory, it is assumed that relativity is a dynamic symmetry, and it is therefore not necessary to introduce it at a "fundamental" level. An equation of this type remains a difficult object to treat. But, making the Hartree-Fock approximation,

$\left\langle\psi_{ \pm}^{\dagger} \psi_{ \pm} \psi_{ \pm}\right\rangle \cong 2 \varphi^{*} \varphi^{2}$

$\left\langle\psi_{\mp}^{\dagger} \psi_{\mp} \psi_{ \pm}\right\rangle \cong \varphi_{\mp}^{*} \varphi_{\mp} \varphi_{ \pm}$,

we get something not less complex, but a very familiar and, above all, conceptually very satisfying connection:

$i \hbar \frac{\partial \varphi}{\partial t}= \pm \frac{\hbar^{2}}{2 m_{p}} \nabla^{2} \varphi_{ \pm} \pm 2 \hbar c V_{p}^{2}\left[2 \varphi_{ \pm}^{*} \varphi_{ \pm}-\varphi_{\mp}^{*} \varphi_{\mp}\right] \varphi_{ \pm}$.

As we know, this is the non-linear GinzburgLandau equation that governs the phenomena of selforganization. In this way, the bases are laid for a possible theory of quantum processes of morphogenesis from the vacuum, hypothesized recently in a slightly different context also by L. Nottale [75]. Reusing the Madelung transformations

$n_{ \pm}=\varphi_{ \pm}^{*} \varphi_{ \pm}$

$n_{ \pm} V_{ \pm}=\mp \frac{i \hbar}{2 m_{p}}\left[\varphi_{ \pm}^{*} \nabla \varphi_{ \pm}-\varphi_{ \pm} \nabla \varphi_{ \pm}^{*}\right]$

Euler equation, and continuity equation for fluids

$\frac{\partial \underline{V}_{ \pm}}{\partial t}+\left(\underline{V}_{ \pm} \nabla\right) \underline{V}_{ \pm}=-\frac{1}{m_{p}} \nabla\left(U_{ \pm}+Q_{ \pm}\right)$,

$\frac{\partial n_{ \pm}}{\partial t}+\nabla\left(n_{ \pm} V_{ \pm}\right)=0$

with $U_{ \pm}=2 m_{p} c^{2} r_{p}^{3}\left(2 n_{ \pm}-n_{\mp}\right)$, we obtains the wellknown Bohm potential [18]:

$Q_{ \pm}=-\frac{\hbar^{2}}{2 m_{p}} \frac{\nabla^{2} \sqrt{n_{ \pm}}}{\sqrt{n_{ \pm}}}$

which can be overlooked for lengths close to that of Planck, while it is a good approximation of the collective behavior in a superfluid space-time, which was recently the subject of a renewed season of studies [76, $77,78]$. On this theoretical system, Winterberg gave the reading and solution of a large number of problems, from the three-dimensionality of the physical space $\mathrm{SO}_{3}$ linked to the group of physical generators $\mathrm{SU}(2)$ (which are, in this case, the two positive and negative modes), a spectrum of masses for hadrons, and leptons up to the dark matter.

Here, according to the aims of the work, we limit ourselves to the emerging nature of QM. It will not escape the reader neither the diversity of styles between 't Hooft and Winterberg, but neither the unavoidable similarities and overlaps. Yet there is an essential difference, which concerns the heart of quantum physics, i.e., the nature of non-locality.

\section{Two Approaches to Non-Locality}

As is known, the non-local effects, tested by experiments such as EPR Bell or GHZ, have a defined range. In general, however, the QM structure does not give indications of a precise limit. For example, the traditional expression of the quantum potential

$Q=-\frac{\hbar^{2}}{2 m} \frac{\nabla^{2} R}{R}$

where $R$ is the amplitude of the wave function, tells us a space-like effect independent of the distance. Yet if we must consider the non-locality something inherent in the space-time, as the locality and determinism of 't Hooft and Winterberg still seem to suggest, then we must necessarily interpret it in terms of dynamic processes in space and time. The problem therefore arises of having a satisfactory physical picture of how, and how much! The correlations in question can resist dissipation and decoherence. In the case of 't Hooft, the central idea is that non-local correlations are a class of equivalence to which local information is subtended which finds its ultimate origin on the Planck scale and hidden by a dissipative mechanism. In other words, something has resisted the separability, overcoming several orders of magnitude and leaving the correlation between two systems A and B intact despite the spatial distance. This something seems to obey a renormalization scheme, which is the characteristic figure of an invariance scale. What is it? This correlation has formed in the typical range of the emergence of QM, when a "wave function" becomes "active", but what has allowed it to maintain itself by exploiting the loss information typical of dissipation? We know that the observables that we use 
in the laboratory can be very different from the "fundamental" beables, so a plausible explanation consists in affirming that two beables connected in the past always remain "below" the observables.

We note that a typical overlap state like "live cat" + "dead cat" has no citizenship in the realm of cellular automata. Imagine that there are two "synchronized" cells C1 and C2 somehow "ancestors" of the overlap, for example: (if $\mathrm{C} 1,2 \mid$ live cat $\rangle \mathrm{C} 2,1 \mid$ dead cat $\rangle$ ), because this ideal synchronization is not only at every moment of the strictly local clock, but, above all, it obeys a formal and not dynamic causality. We must therefore admit that the $\mathrm{AC}$ interpretation is a toy model. In order to acquire the physical consistency, we must make assumptions about the dynamics of the cells (and the nature of the beables!). For example, using oscillators, as in [39]. We therefore expect to find the non-locality as a form of the coherence of these oscillators and the prediction of a precise correlation limit. This problem is still open and very complex and is the main chapter of a unification between $\mathrm{QM}$ and QFT which is still to be written. In fact, most of the QFTs have developed under the impulse of particle physics and high energies, while the traditional treatments of non-locality are located at low energies [79].

In Wintenberg's theory, the vacuum is a ZPF (Zero Point Fluctuation) model triggered by the dynamics between positive and negative modes. The ZPF issue, too, as an "interface" between the QM vacuum and the QFT multiple-state one is highly debated [80]. Denoting the wave number by $k$, Wintenberg makes the "simple" hypothesis that the trend of the zero-point energy spectrum is given by

$f(k)=\hbar c k^{3}$.

Thus, the energetic spectrum of the turbulence obeys the equation

$F(k)=$ const $k^{-5 / 3}$.

On these bases, Winterberg arrives at a "generous" prediction for the breaking of the correlations over about $100 \mathrm{~km}$, evidently compatible with all the experiments carried out so far. Recently Gkiouleka [81] has taken over and refined the Winterberg hypotheses and halved the forecast.

A part of the mystery is that we don't really understand what the wave function really is. Winterberg assumes that the wave function is a genuine physical field that really collapses. In the same paper, he also reviews the early literature on the subject. It is hard to accept this viewpoint and not expect the collapse to propagate at a finite speed, or to not be disrupted by a possible noise in the mechanism that propagates it. From the stand-point of the Bohmian interpretation, the wave function of the combined physical system and the measuring apparatus never really collapses! Nonetheless, even in the Bohmian interpretation, one models (instead of deriving from first principles) the Hamiltonian governing the interaction between a system and an apparatus during the measurement. The model is just a model, and there is no need for it to be exact. Furthermore, one can expect a breakdown in quantum correlations, if there is a small amount of noise, presumably from subquantum processes, in the guidance condition that determines the particle velocities from the wave function (Gkiouleka, 2008).

In both 't Hooft's and Winterberg's theories, in fact, the wave function is emerging and is therefore a real physical object that describes collective behaviors under dissipation. The coarse-grained existence is still an existence! This should lead to an objective theory of collapse as a break of the coherence, which is expected at finite speeds, because, in any case, the range of quantum manifestations is bound by relativistic symmetries. In fact, both authors did not escape this request and proposed an explanation $[82,83]$. The question of the breakdown of correlations passes to future experiments in optics and quantum information, but the impression remains that the non-locality falls into difficulty in a traditional dynamic vision, as Heisenberg had already claimed [84]. In this direction, we find that the "synchronic" approaches typical of the last Bohm one and the more recent ones based on the time-reversal have recently been taken up by different groups with different nuances. It seems that there cannot be any easy "peaceful coexistence" between Relativity and QM, even under the hypothesis that both are emerging [85-87]. As N. Gisin and A. Suarez said, the non-locality seems "something is coming from the outside space and time". In relation to these two authors, the recurrent debate on the determinism and free will should be briefly mentioned here, in which 't Hooft [88-90] participated actively. In a nutshell, the matter is this. In the EPR-Bell experiment, it is crucial that one of the 
experimenters - according to quantum information conventions, we will call it Bob - can choose how to orient the detector, whether to measure spin up or spin down independently of the other experimenter that is traditionally Alice. For some reason, it is believed that the reintroduction of some form of determinism and subquantum locality must also have an effect on the brains of the experimenters. Therefore, since there are no actual physical influences between Alice and Bob, the brains of both must be correlated and synchronized like the beables in play in the experiment. Even those who try to use more traditional schemes than 't Hooft cannot escape the temptation to add a piece to the question, as can be seen from this suffered Suarez passage:

I would like to stress that it is not sufficient to assume "free will" in order to escape "many worlds" or "parallel lives." One has to reject "empty waves" as well and therefore to accept that decision of outcome happens at detection. My argument is as follows: If we accept that nonlocal coordination of outcomes is in principle possible, by which particular reason should we then reject nonlocal decision at detection? Only because we assume that the outputs of devices are necessarily determined by some cause in the past light-cone. But then one must consequently also assume that the outputs of the experimenter's brain are predetermined, and therefore he has no free will. In other words, the three assumptions: free will, "empty waves" and non locality cannot hold together. And this means that for the sake of free will assuming "empty waves", and therefore "many worlds" and "parallel lives", is not better than assuming Gerard 't Hooft's superdeterminism (Suarez, 2012).

It is difficult to understand how the traditional epistemological battles of QM can be extended to the human brain. Inside our head, there is certainly ample room for "hidden variables" of the cognitive type linked to the specific methods of biological processing of data, but it is not absolutely necessary that they have to do with quantum beables. We have already said that the behaviors of an emerging level are not necessarily linked to those of the lower level, and indeed they can take control over it, becoming, in fact, autonomous. Superdeterminism, on the other hand, crosses completely different ranges and dynamics. It seems, therefore, as Hawking repeatedly said with the usual irony, "an attempt to solve a mystery with another mystery". There is also a more physical reason to distance ourselves from the setting of this debate. We have seen that a possible future physicist of the 't Hooft equivalence classes is to study them in terms of oscillator coherence. In this case, there is a physical connection between Alice and Bob, and it is not necessary to postulate an invertible and "subtle" correlation in the past between their brains and their "free" choices.

In conclusion, we want to mention a case doubly interesting from the physical-mathematical and sociological points of view, which still concerns Winterberg. In fact, in 2013, the scholar proposed a new topological version of the EPR-Bell correlations, but the central idea became famous, however, with the abbreviation ER $=$ EPR (Einstein-Rosen Bridge $=$ $=\mathrm{EPR}$ ) used in the publications by Maldacena and Susskind [91, 92]. Indeed, the two proposals are linked to different contexts, even if a strong underlying conceptual analogy can be certainly identified. One starts from a Euclidean substrate, where the ordinary space-time has not yet emerged, and, therefore, the signature of the metric is of the type $(+,+,+,+)$. It is assumed that a whormhole can connect two distant places that they are therefore "entangled" within space and time. Euclidean approaches are of fundamental importance in quantum cosmology to characterize the early stages of the "Big Bang" [93, 94, 95]. Using an effective image is like joining two distant points on a piece of paper bypassing the surface with a "bridge" from the outside. The idea of Susskind and Maldacena was born in the context of the holographic principle and black holes, while the work of Wintenberg uses the mathematics of the complex surfaces developed by P. Teichmüller, a young German mathematician who died prematurely on the Russian front (1913-1943) and recently brought to the attention of the mathematical community by the works of Shinichi Mochizuki. A detailed exposition of these approaches goes beyond the scope of this work. In terms of Planck's cells, these works suggest two important things: 1) Planck's scale should not be seen as the hic sunt leones of physics, but rather of "ordinary" space-time, and 2) a quantum system that is formed near the edge of a Planck cell is already strongly correlated. In particular, the work of Susskind and Maldacena is a part of the series of proposals that place a non-local correlation at the very origin of the spacetime [96-98]. One of the emerging frontiers of theoretical physics is the search for a synthesis between new 
foundational tendencies and particle physics. Here, it seems clear the difference between a line like the one analyzed in this paper, the 't Hooft or Winterberg, which is traditionally dynamic, even if pushed to the Planck scale, and other works that call into question the concept of time in the quantum realm and extend the emergency to the same space-temporal structure [98]. Some of these go in the direction of a convergence between the holography and the emergent space-time [99-102].

\section{Conclusions}

At the end of this rapid journey on primitive quantization theories, it is legitimate to ask what the two great contenders, Einstein and Bohr, would have said. Einstein would have appreciated the return of some form of locality and determinism, albeit to the elusive Planck scale, and it is possible that the nature of the non-local correlations remains for a long time still well hidden behind noise and dissipation. On the other hand, Bohr's "impregnable" position appears to be dated, similar to the story of the six blind men attempting to describe an elephant through measurements. Non-locality is no longer an "unexpected guest" and the current contrast between dynamic theories and time reversal shows rather that the old debate on the locality is progressively transforming into the one of the emergency of locality in time, which is then common to the challenges beyond the Standard Model and toward quantum gravity.

1. J.S. Bell. On the Einstein Podolsky Rosen Paradox. Physics 1, 195 (1964).

2. G. Ghirardi, R. Romano. Is a description deeper than the quantum one possible? Phys. Scr. T 163, 014028 (2014).

3. G. ' $\mathrm{t}$ Hooft. Dimensional Reduction in Quantum Gravity. arXiv:gr-qc/9310026 (1993).

4. Y.J. Ng, H. Van Dam. Space time foam, holographic principle, and black hole quantum computer. Int. J. Mod. Phys. A 20, 1328 (2005).

5. I. Licata, D. Fiscaletti. Bohm trajectories and Feynman paths in light of quantum entropy. Act. Phys. Pol. B 45 (4), 885 (2014).

6. E.R. Caianello. Quantum systems and other physics as systems theory. Riv. N. Cim. 15, 4 (1992).

7. H.J. Treder, H.H. von Borzeszkowski. The Meaning of Quantum Gravity (Reidel, 1988).

8. J.A. Wheeler. Information, physics, quantum: The search for links. In: W.H. Zurek (Ed.), Complexity, Entropy, and the Physics of Information (Addison-Wesley, 1990).
9. I. Licata, A. Sakaji(Eds.), Physics of Emergence and Organization (World Scientific, 2008).

10. J. Busemeyer, P. Bruza. Quantum Models of Cognition and Decision (Cambridge Univ. Press, 2012).

11. A.I. Khrennikov. Ubiquitous Quantum Structures: From Psychology to Finance (Springer, 2010).

12. K. Kitto. A contextualised general systems theory. Systems 2, 541 (2014)

13. L. Gabora, D. Aerts. Contextualizing concepts using a mathematical generalization of the quantum formalism. Jour. Exp. Theor. Artificial Intelligence 14 (4), 327 (2002).

14. I. Licata. General system theory, like-quantum semantics and fuzzy sets. In: G. Minati, E. Pessa, M. Abram (Eds.), Systemics of Emergence. Research and Development (Springer, 2006).

15. H. Atmanspacher. Contextual emergence from physics to cognitive neuroscience. Jour. Conscious. Stud. 14, 18 (2007).

16. N.A. Baas, C. Emmeche. On emergence and explanation. Intellectica 25, 67 (1997).

17. D. Bohm, B.J. Hiley. The Undivided Universe: An Ontological Interpretation of Quantum Theory (Routledge, 1993).

18. I. Licata, D. Fiscaletti. Quantum Potential: Physics, Geometry and Algebra (Springer, 2014).

19. H. Atmanspacher, H. Römer, H. Walach. Weak quantum theory: complementarity and entanglement in physics and beyond. Found. Phys. 32 (3), 379 (2002).

20. H. Atmanspacher, P. bein Graben, T. Filk. Can classical epistemic states be entangled? D. Song, M. Melucci, I. Frommholz, P. Zhang, L. Wang, S. Arafat (Eds.) Quantum Interaction (Springer, 2011).

21. F. Heylighen. Classical and non-classical representations in physics I. Cybernetics and Systems 21, 423 (1990).

22. S.B. Kuksin, A.I. Neishtadt. On quantum averaging, quantum KAM, and quantum diffusion. Russ. Math. Survey 68 (2), 335 (2013).

23. W.C. McHarris. Chaos and the quantum: How nonlinear effects can explain certain quantum paradoxes. Jour. Phys.: Conf. Ser. 306, 012050 (2011).

24. W. Xiao-Qian, M. Jian, Z. Xi-He, W. Xiao-Guang. Chaos and quantum Fisher information in the quantum kicked top. Chin. Phys. B 20 (5), 050510 (2011).

25. G. Resconi, I. Licata, D. Fiscaletti. Unification of quantum and gravity by non classical information entropy space. Entropy 15 (9), 3602 (2013).

26. I. Licata, D. Fiscaletti. Weyl geometries, Fisher information and quantum entropy in quantum mechanics. Int. J. Theor. Phys. 51 (11), 3587 (2012).

27. I. Licata. Why the collective behavior of classic neurons is so well approximated by a quantum potential? $A d v$. Sc., Eng. Med. 6, 1 (2014).

28. G. 't Hooft. Equivalence relations between deterministic and quantum mechanical systems. J. Stat. Phys. 53, 323 (1988).

ISSN 2071-0194. Ukr. J. Phys. 2020. Vol. 65, No. 1 
29. G. 't Hooft. How a wave function can collapse without violating Schr'odinger's equation, and how to understand Born's rule. arXiv: 1112.1811 [quant-ph] (2012).

30. G. 't Hooft. The fate of the quantum. arXiv:1308.1007 [quant-ph] (2013).

31. G. 't Hooft. The cellular automaton interpretation of quantum mechanics. A view on the quantum nature of our Universe, compulsory or impossible? ArXiv:1405.1548 [quant-ph] (2014).

32. S. Wolfram. A New Kind of Science (Wolfram Media, 2002).

33. G. t' Hooft. Does God play dice? Phys. World (December, 2005).

34. G.'t Hooft. Quantum mechanics from classical logic. J. Phys.: Conf. Ser. 361, 012024 (2012).

35. X.F. Liu, C.P. Sun, Consequences of 't Hooft's equivalence class theory and symmetry by coarse graining. J. Math. Phys. 42 (8), 3665 (2001).

36. M. Blasone, P. Jizba, G. Vitiello. Dissipation and quantization. Phys. Lett. A 286 (3/4), 205 (2001).

37. M. Blasone, P. Jizba, H. Kleinert. 't Hooft's quantum determinism - path integral viewpoint. Braz. J. Phys. 35 (2B), 497 (2005).

38. H.T. Elze. Deterministic models of quantum fields. J. Phys.: Conf. Ser. 33, 399 (2006).

39. P. Jizba, F. Scardigli, M. Blasone, G. Vitiello. 't Hooft quantization for interacting systems. J. Phys.: Conf. Ser. 343, 012110 (2012).

40. R. Gambini, J. Pullin. Holography from loop quantum gravity. Int. J. Mod. Phys. D 17 (3/4), 545 (2008).

41. J.N. Ng. Spacetime foam: from entropy and holography to infinite statistics and non-locality. Entropy 10, 441 (2008).

42. J. Magueijo, L. Smolin. Gravity's rainbow. Class. Quant. Grav. 21 (7), 1725 (2004).

43. G.'t Hooft. The mathematical basis for deterministic quantum mechanics. J. Phys. Conf. Ser. 67, 012015 (2007).

44. T.H. Elze. Note on the existence theorem in "emergent quantum mechanics and emergent symmetries". J. Phys. A: Math. Theor. 41, 304020 (2008).

45. D. Dolce. Elementary spacetime cycles. EPL 102 (3), 31002 (2013)

46. A. Zeilinger. Dance of the Photons: From Einstein to Quantum Teleportation (Farrar, Straus, and Giroux, 2010).

47. J.A. Larsson. Loopholes in Bell inequality tests of local realism. J. Phys. A: Math. Theor. 47, 424003 (2014).

48. W. Heisenberg. Introduction to the Unified Field Theory of Elementary Particles (Interscience, 1966).

49. O. Freire. The Quantum Dissidents. Rebuilding the Foundations of Quantum Mechanics (1950-1990) (Springer, 2015).

50. G. Amelino-Camelia. Doubly-special relativity: facts, myths and some key open issues. Symmetry 2, 230 (2010).

51. F. Winterberg. Low energy consequences of high-energy quantum chaos. Int. J. Theor. Phys. 31 (8), 1375 (1992).
52. F. Winterberg. Physical continuum and the problem of a finitistic quantum field theory. Int. J. Theor. Phys. 32 (2), 261 (1993).

53. F. Winterberg. Hierarchical order of Galilei and Lorentz invariance in the structure of matter. Int. J. Theor. Phys. 32 (9), 1549 (1993).

54. F. Winterberg. Equivalence and gauge in the Planck-scale aether model. Int. J. Theor. Phys. 34 (2), 265 (1995).

55. F. Winterberg. Derivation of quantum mechanics from the Boltzmann equation for the Planck aether. Int. J. Theor. Phys. 34 (1), 2145 (1995).

56. F. Winterberg. Conjectured breaking of the superluminal quantum correlations by turbulent fluctuations of the zero point vacuum field. Z. Naturforsch. 53a, 659 (1998).

57. F. Winterberg. Planck mass plasma vacuum conjecture. Z. Naturforsch 58a, 231 (2003).

58. F. Winterberg. Relativistic quantum mechanics as a consequence of the Planck mass plasma conjecture. Int. J. Theor. Phys. 46 (12), 3294 (2007).

59. L. Janossy. A new approach to the theory of relativity. III. Problem of the ether. Found. Phys. 2 (1), 9 (1972).

60. L. Kostro. The physical meaning of Albert Einstein's relativistic ether concept. In: Frontiers of Fundamental Physics. Edited by F. Barone, F. Selleri (Springer, 1994), pp. 193-201.

61. D. Meschini, M. Letho. Is empty spacetime a physical thing? Found. Phys. 36 (8), 1193 (2006).

62. H. Bondi. Negative mass in general relativity. Rev. Mod. Phys. 29 (3), 423 (1957).

63. W.B. Bonnor. Negative mass in general relativity. General Relativity and Gravitation 21 (11), 1143 (1989).

64. A.D. Sakharov. Vacuum quantum fluctuations in curved space and the theory of gravitation. Sov. Phys. - Doklady 12, 1040 (1968)].

65. B.L. Hu. General Relativity as geometro-hydrodynamics, arXiv:gr-qc/9607070 (1996).

66. A.D. Sakharov. Cosmological model of the Universe with a time vector inversion. JETP Lett. 52, 349 (1980).

67. L. Marochnik, D. Usikov. Inflation and CMB anisotropy from quantum metric fluctuations. Grav. Cosm. 21 (2), 118 (2015).

68. J. Pecina-Cruz. Time reversal induces negative mass and charge conjugation: On the physical interpretation of the irreducible unitary representations of negative mass and energy of the full poincare group. arXiv:hep-ph/0505188 (2005).

69. J. Belletête, M. Paranjape. On negative mass. Int. J. Mod. Phys. D 22, 1341017 (2013).

70. M. Saoussen, M. Paranjape. Negative mass bubbles in de Sitter spacetime. Phys. Rev. D 90, 101502 (2014).

71. L. Chiatti, I. Licata. Relativity with respect to measurement: Collapse and quantum events from fock to cramer. Systems 2 (4), 576 (2014).

72. D. Hestenes. The zitterbewegung interpretation of quantum mechanics. Found. Phys. 20 (10), 1213 (1990). 
73. L. de la Pena, A.M. Cetto, A. Valdes-Hernandez. The Emerging Quantum. The Physics behind Quantum Mechanics (Springer, 2015).

74. M.P. Davidson. A generalization of the Fenyes-Nelson stochastic model of quantum mechanics. Lett. Math. Phys. 3, 271 (1979).

75. L. Nottale. Generalized quantum potentials. Jour. Phys. A: Math. Theor. 42 (27), 275306 (2009).

76. E. Di Casola, S. Liberati, S. Sonego. Between quantum and classical gravity: Is there a mesoscopic spacetime? Found. Phys. 45 (2), 171 (2015).

77. B.L. Hu. Can spacetime be a condensate? Int. J. Theor. Phys. 44 (10), 1785 (2005)

78. M. Consoli, M. Probing the vacuum of particle physics with precise laser interferometry. Found. Phys. 45 (1), 22 (2015).

79. A.O. Barvinsky. Aspects of nonlocality in quantum field theory, quantum gravity and cosmology. arXiv:1408.6112 [hep-th] (2014).

80. P.C.W. Davies. Quantum vacuum noise in physics and cosmology. Chaos 11 (3), 539 (2001).

81. E. Gkioulekas. Winterberg's conjectured breaking of the superluminal quantum correlations over large distances. Int. J. Theor. Phys. 47 (5), 1195 (2008).

82. G. 't Hooft. How a wave function can collapse without violating Schr'odinger's equation, and how to understand Born's rule. arXiv:1112.1811 [quant-ph] (2011).

83. F. Winterberg. Wave function collapse as a real physical phenomenon caused by vacuum fluctuations near the Planck scale. Z. Naturforsch 46a, 746 (1991).

84. W. Heisenberg. The Physical Principles of the Quantum Theory (Dover, 1998).

85. I. Licata, L. Chiatti. Timeless approach to quantum jumps. Quanta 4, 1 (2015).

86. M. Silberstein, W.M. Stuckey, T. McDevitt. Being, becoming and the undivided universe: A dialogue between relational blockworld and the implicate order concerning the unification of relativity and quantum theory. Found. Phys. 43 (4), 502 (2013).

87. R. Kastner. The Transactional Interpretation of Quantum Mechanics (Cambridge Univ. Press, 2013).

88. A. Suarez, P. Adams (eds) Is Science Compatible with Free Will? Exploring Free Will and Consciousness in the Light of Quantum Physics and Neuroscience (Springer, 2013).

89. A. Suarez. Empty waves, many worlds, parallel lives, and nonlocal decision at detection. arXiv:1204.1732 [quant-ph] (2012).
90. G.'t Hooft. The free-will postulate in quantum mechanics. arXiv:quant-ph/0701097 (2007).

91. F. Winterberg. Teichmüller space interpretation of quantum mechanics. Ann. Fond. Louis de Broglie 38, 129 (2013).

92. L. Maldacena, L. Susskind. Cool horizons for entangled black holes. Fortschr. Phys. 61, 781 (2013).

93. J.B. Hartle, S.W. Hawking. Wave function of the Universe. Phys. Rev. D 28, 2960-2975 (1983).

94. I. Licata. A Note on the origin of time in archaic universe. NeuroQuant. 12 (1), 126 (2014).

95. F. Feleppa, I. Licata, C. Corda. Hartle-Hawking boundary conditions as nucleation by de Sitter vacuum. Phys. Dark. Un. 26, 100381 (2019).

96. M. van Raamsdonk. Building up spacetime with quantum entanglement. Gen. Rel. Grav. 42, 2323 (2010).

97. F. Markopoulou. Space does not exist, so time can. arXiv:0909.1861 [gr-qc] (2008)

98. T.P. Singh. Space-time from collapse of the wave-function. Z. Naturforsch. A 74, 147 (2019).

99. L. Chiatti, I. Licata. Particle model from quantum foundations. Quantum Stud.: Math. Found. 4 (2), 181 (2017).

100. I. Licata, L. Chiatti. Event-based quantum mechanics: A context for the emergence of classical information. Symmetry 11 (2), 181 (2019).

101. X. Dong, E. Silverstein, G. Torroba. De Sitter holography and entanglement entropy. J. High Energ. Phys. 7, 50 (2018).

102. T. Vistarini. Holographic space and time: Emergent in what sense? Studies Hist. Phil. Mod. Phys. 59, 126-135 (2017).

Received 16.03.19

\section{I. Лiкama}

\section{IНТЕРПРЕТАЦЯ КВАНТОВОї}

\section{МЕХАНІКИ НА ПЛАНКІВСЬКОМУ МАСШТАБІ}

$\mathrm{P}$ е $з$ ю м е

В останні роки було створено багато різних теорій первинного квантування на планківському масштабі. Їх метою була розробка моделі вакууму, здатної обгрунтувати дослідження за рамками Стандартної Моделі. Хоча ця мета претензійна і орієнтована на фізику частинок, їі необхідним помітним наслідком є можливість розглянути квантову механіку по-новому. Можливі різні гіпотези про елементарні осередки. Ми концентруємося на концептуальних рисах теорій Г. 'т Хоофта і Ф. Вінтерберга, приділяючи увагу виникненню нелокальних кореляцій. Ці теорії створюють новий стиль в інтерпретації квантової механіки. 